\title{
Superfluidity Nature of A Rotating Bose-Einstein Condensation with Finite Size Effect
}

\author{
Alyaa A. Mahmoud*, M. M. Salah and S. S. M. Soliman \\ Department of Physics, Faculty of Science, Minia University, El Minia, Egypt
}

\begin{abstract}
W E report on a superfluid properties of a Bose- Einstein condensate in a rotating harmonic trap. We employ the semiclassical approximation, which is the density of state approach. Our approach provided the finite size effect. The superfluid fraction of bosons and its heat capacity at constant volume are investigated. The calculated results provide a good qualitative agreement with the measured experimental results for superfluid helium ${ }^{4} \mathrm{H}$. Moreover, furnish useful qualitatively theoretical results for a spectroscopic measurement of the superfluid fraction of an ultracold atomic gas [N. R. Cooper and Z. Hadzibabic, Phys. Rev. Lett. 104, 030401 (2010)]. Our results show that both The superfluid fraction of bosons and its heat capacity depend on the number of the atoms. This significant dependence should be considered when the it in situ widths are used in extracting measurable experimental quantities. Our approach can be extended to consider the rotating condensate in optical potential .
\end{abstract}

\section{Introduction}

Ultracold atomic gases, in a state of BoseEinstein condensation (BEC), provide a highly controllable experimental setting for studies of many-body quantum phenomena, in particular superuidity and superconductivity [1]. Both of these two phenomena may occur in a systems which obey Bose-statistics. In the Bose-systems such a condensate appears due to a direct grouping of bosons in the ground state and in the Fermi systems due to the formation of Cooper pairs, which are also bosons. Therefore, it seems very attractive to explain superfluidity of the BEC.

Experimentally, superfluidity nature of an ultracold boson gases in a state of BEC has become a subject of extensive investigation. In this sprite, Cooper and Hadzibabic [2] proposed a direct method to measure the superfluid fraction of an ultra cold atomic boson gas. This method is closely analogous to the classic experimental method of Andronikashvili [3, 4] for measuring the superfluid fraction of heluim liquid. The key idea in experiments is that the super fluid is distinguished from normal fluid by their unusual response to rotation. However, a natural way to discuss superfluidity in a BEC system is to focus on its rotational properties. For a macroscopic system the moment of inertia is given by the rigid value unless the condensate exhibits superfluidity. Crucial deviations from the rigid motion occur in rotating liquid helium below the lambda temperature (transition temperature from normal helium to superfluid helium, heluim II ).

Superfluid phase of He II possesses some more unique properties such as: (i) The superfluid fraction can be defined via the response of the system to infinitesimal rotations. The superfluid part shows no response to rotations at all while its density distribution contributes to the classical moment of inertia. (ii) the shape of the specific heat capacity versus temperature curve resembles the shape of the letter $\boldsymbol{\lambda}$ and therefore, the singular point on this curve is called $\boldsymbol{\lambda}$ point. The specific heat diverge logarithmically at $\boldsymbol{\lambda}$ transition, it increases sharply at temperature less than $T \boldsymbol{\lambda}$ until it shows a steep maximum at about $2.17 \mathrm{~K}$ followed by a sharp fall at $T>2.17 \mathrm{~K}$ to a shallow minimum at $2.6 \mathrm{~K}$, beyond which it again increases slowly. So, if we want to investigate the physical mechanisms of the superfluidity of BEC we should calculate these two parameters.

In this respect, Stringari [5] drew a parallel between the rotating BEC and the superfluid systems, and he pointed out that the rotational properties of a BEC provides a natural way to analyze the deviations from a rigid motion due to condensation. Several studies showed that the evidence of the superfluidity in a rotating BEC is the reduction of the moment of inertia below the classical rigid-body value [6 -10]. The reduction in superfluid density depresses the transition temperature and is expected to cause the specific 
heat capacity to be enhanced. However, BEC is usually accompanied by a cusp in the temperature dependence of the heat capacity $[11,12,13,14]$. The essential features of the superfluidity of BEC are clearly exhibited in the behavior of the specific heat, such as in the case of the _-point superfluid transition of liquid helium, which is observed in its heat capacity.

In this paper we present a calculation of the superfluid fraction and the heat capacity for a cloud of non-interacting BEC in a rotating trap. The semi classical approximation, which is the density of state (DOS) approach, is employed to calculate the thermodynamic potential. Finite size effects are allowed for by calculating the appropriate DOS for this regime. Using the thermodynamical potential, the transition temperature, superfluid fraction and the heat capacity are calculated. Our calculations are complementary to [5] and show markedly different results for the superfluid fraction. The

$V_{(x, y, z)}=\frac{1}{2} m\left[\omega_{x} x^{2}+\omega_{y} y^{2}+\omega_{z} z^{2}\right]$

where $\boldsymbol{\omega}_{\boldsymbol{x}}, \boldsymbol{\omega}_{\boldsymbol{y}}$ and $\boldsymbol{\omega}_{\boldsymbol{z}}$ are the trapping frequencies, respectively, and $m$ is the atomic mass. In the presence of an uniformly rigid obtained numerical results are calculated by using the trap parameters of Abo-Shaeer et al. experiment [Abo-Shaeer] and qualitatively compared with the experimental data of He II.

The calculated results showed that the superfluid fraction is independent on the rotation rate while the heat capacity is dependent strongly on the rotation rate for the whole temperature range. Both of them depend on the finite size.

The paper is planned as follows: section two includes the system definition and a systematic method for calculating the accurate thermodynamic potential. The superfluid fraction is given in section three. In section four, the heat capacity is given. Conclusion is given in the last section.

\section{Basic formalism}

Our model consists of $\mathrm{N}$ particles living in a exterior potential

rotation in the $x y$-plane with an angular velocity $\boldsymbol{\Omega}\left(\equiv \Omega_{\hat{e}_{z}}\right)$ around the $z$-axis, the Hamiltonian describing the system is given by [15]

$$
H_{\Omega}=\frac{P_{x}^{2}+P_{y}^{2}+P_{z}^{2}}{2 m}+\frac{1}{2} m\left[\omega_{x} x^{2}+\omega_{y} y^{2}+\omega_{z} z^{2}\right]-\Omega L_{z}
$$

where $L_{z}$ is the angular momentum $L_{z}=x p_{y}-y p_{x}$. Hamiltonian in Eq.(2) is

$$
\epsilon_{n, n . n}=n_{+} \hbar\left(\omega_{x}-\Omega\right)+n_{-} \hbar\left(\omega_{x}-\Omega\right)+n \hbar \omega_{z}+\epsilon_{0.0 .0}
$$
$[13,16,17]$ given by

trapped gas in (2), requires that the rotation rate is limited to:

For $\boldsymbol{\Omega}=\boldsymbol{\omega}_{\boldsymbol{x}}=\boldsymbol{\omega}_{\boldsymbol{y}} \equiv \boldsymbol{\omega}_{\perp}$ the Hamiltonian the ground state energy and $n_{+}, n$ and $n$ are nonnegative integers. Stability of the harmonically

$$
\Omega<\omega_{x}
$$

(2) describes a quasi-2D system of particles in a uniform magnetic field. The energy spectrum

$$
\epsilon_{n_{-}, n}=2 n_{-} \hbar+\left(\omega_{\perp}+\frac{1}{2}\right)+n \hbar\left(\omega_{z}+\frac{1}{2}\right)
$$

takes the form familiar from studies of the quantum Hall effect,
Where $\boldsymbol{n}_{-}=0,1,2, \ldots$ is the Landau level index $, m=-n_{-},-n_{-}+1,-n_{-}+2, \ldots$. the angular momentum quantum number about the rotation axis, and $\boldsymbol{n}_{\boldsymbol{z}}=\mathbf{1}, \mathbf{2}, \ldots$ is the sub band index for motion along the rotation axis. The spectrum is highly degenerate, with the single particle states of different angular momentum $\mathrm{m}$ having the same energy. In the following we will consider a system characterized by the condition Eq.(4) for any $\omega_{y}$. 
Usually BEC is described within the grand canonical ensemble. Once $\mu$ has been determined, all relevant thermodynamical quantities can be calculated from partial derivative of the

$$
q=-\sum_{n_{+}, n_{-}, n} \ln \left(1-z e^{-\beta \epsilon_{n_{+}, n_{-}, n}}\right)
$$

where $\beta=1 / k_{\beta} T, z=e^{\beta\left(\mu-\epsilon_{0,0,0}\right)}$ is the fugacity. In order to use the semiclassical approximation, summation over $i$ is converted into integral weighted by an accurate DOS $\rho(\epsilon)$

it is convenient to expand the logarithm in

$$
\begin{aligned}
q=q_{0}+\sum_{n_{+}, n_{-}, n}^{\infty} \frac{z e^{-\beta \epsilon_{n_{+}, n_{-}, n}}}{1-z e^{-\beta \epsilon_{n_{+}, n_{-}, n}}} & \\
& =q_{0}+\sum_{j=1}^{\infty} \frac{z^{j}}{j} \int_{0}^{\infty} \rho(\epsilon) e^{-j \beta \epsilon} d \epsilon
\end{aligned}
$$

approximated formula for the DOS is given by where $q_{0}=-g_{0} \ln \left(1-z e^{-\beta \epsilon_{0,0,0}}\right)$. An [13]

$$
\rho(\epsilon)=\frac{1}{\alpha}\left\{\frac{1}{2} \frac{\epsilon^{2}}{\left(\hbar \omega_{g}\right)^{3}}+\frac{3}{2}\left(\frac{\varpi}{w_{g}}\right) \frac{\epsilon}{\left(\hbar \omega_{g}\right)^{2}}+\frac{\left(9 \varpi^{2}-\varpi_{r}^{2}\right)}{8 \hbar w_{g}^{2}}\right\}
$$

Where $\omega_{g}=\left(\omega_{x} \omega_{y} \omega_{z}\right)^{1 / 3}, \varpi=\left(\omega_{x}+\omega_{y}+\omega_{z}\right) / 3$ and

$$
\alpha=\left(1-\frac{\Omega}{\omega_{x}}\right)\left(1+\frac{\Omega}{\omega_{y}}\right)
$$

In Eq.(8) the last term is a constant term, it is a function in the oscillator frequencies only. However, this term will produce $\zeta$ (1) function in the condensation fraction $\frac{N}{N_{1}}$ and BEC transition temperature $T_{o}$. Since $\zeta(1)=\infty$ then $N_{0}$ and $\frac{N}{N_{0}}$ are undefined at the onset of the condensation. In the following, we will drop the contribution from this term. For the non- rotating ideal Bose gas trapped in an isotropic potential with frequency $\omega$ the calculated DOS in Ref's $[12,14]$ is recovered.

$$
\rho(\epsilon)=\frac{1}{2} \frac{\epsilon^{2}}{(\hbar \omega)^{3}}+\frac{3}{2}\left(\frac{\varpi}{w_{g}}\right) \frac{\epsilon}{(\hbar \omega)^{2}}+\frac{1}{\hbar \omega}
$$

Expand the logarithm in eq.6 and using semiclassical approximation . Substituting Eq.(8) in Eq .(7) we have the thermodynamical potential for the confined Bose gas in a rotating trap,

$$
q=q_{0}+\frac{1}{\alpha}\left\{\frac{1}{2}\left(\frac{K_{\beta} T}{\hbar \omega_{g}}\right)^{3} g_{4}(z)+\frac{3}{2}\left(\frac{\varpi}{w_{g}}\right)\left(\frac{K_{\beta} T}{\hbar \omega_{g}}\right)^{2} g_{3}(z)\right\}
$$

where $\mathrm{g}_{\mathrm{k}}(z)=-\sum_{j=1}^{\infty} z^{j} / j^{k}$ is the usual Bose function. 
Superfluidity of condensate boson in a rotating harmonic trap

Transition temperature

In order to improve the agreement between the superfluid Helium II and the superfluidity nature

$$
\begin{gathered}
N=\left(\frac{\partial q}{\partial z}\right)_{T} \\
=N_{O}+\frac{1}{\alpha}\left\{\frac{1}{2}\left(\frac{K_{\beta} T}{\hbar \omega_{g}}\right)^{3} g_{3}(z)+\frac{3}{2}\left(\frac{\varpi}{w_{g}}\right)\left(\frac{K_{\beta} T}{\hbar \omega_{g}}\right)^{2} g_{2}(z)\right\}
\end{gathered}
$$

The condensate transition temperature with theonset of condensation, $N_{0}=0$ and we have, finite size effect is calculated from Eq.(11). At

$$
T_{c}(\alpha)=T_{0}\left[1-\frac{1}{2} \frac{\varpi}{w_{g}} \frac{\zeta(2)}{\zeta(3)}\left(\frac{\zeta(3)}{N}\right)^{1 / 3}\right] \alpha^{1 / 3}
$$

Where

$$
T_{0}=\frac{\hbar \omega_{g}}{K_{\beta}}\left(\frac{N}{\zeta(3)}\right)^{1 / 3}
$$

is the ideal BEC transition temperature, and $\zeta$ is the Riemann zeta function. The second term in Eq.(12) gives the finite size correction to the ideal gas result .

$$
\frac{\rho_{s}}{\rho}=1-\frac{\theta}{\theta_{\text {rig }}}
$$

where $\theta_{\text {rig }}$ is the classical moment of inertia for a cloud rotate as a rigid body and $\theta$ is the

$$
\theta_{\text {rig }}=m N\left(x^{2}+y^{2}\right)
$$

The moment of inertia of the condensate bosons (super-fluid phase) is determined from the

$$
\left\langle L_{z}\right\rangle=\Omega \theta
$$

where the average here is taken over the state perturbed by Hext. Following Dalvofo et al. [34],

the moment of inertia $\theta$, relative to the $z$-axis,

\section{superfluid fraction}

The definition of superfluid fraction of an atomic cloud can be formulated in a formal and quantitative way using the cloud's response to a rotation of the external potential, i.e. the moment of inertia [7, 19, 20],

observed moment of inertia of the condensed bosons. For a rigid body rotation, the moment of inertia is given by the value

quantum-mechanical arguments. In this case $\theta$ is given by [21],

can be defined as the linear response of the system to a rotational field $H_{e x t}=-\Omega L z$, according to the formula

$$
\begin{aligned}
\theta & =\frac{2}{z} \sum_{i, j} \frac{\left|\left\langle j\left|L_{z}\right| i\right\rangle\right|^{2}}{E_{i}-E_{j}} e^{-\beta E_{j}} \\
& =\frac{m N}{\left(\omega_{x}^{2}-\omega_{y}^{2}\right)}\left[\left(\left\langle y^{2}\right\rangle-\left\langle x^{2}\right\rangle\right)\left(\omega_{x}^{2}+\omega_{y}^{2}\right)-2\left(\omega_{y}^{2}\left\langle y^{2}\right\rangle-\omega_{x}^{2}\left\langle x^{2}\right\rangle\right)\right]
\end{aligned}
$$


Equations $(15,17)$ are exact results for the Hamiltonian (2) and could be applied to any value of particle number $N$. For the explicit evaluation of the moment of inertia in a Bose gas it is useful to evaluate the average In situ radii $\left\langle x^{2}\right\rangle$ and $\left\langle y^{2}\right\rangle$ in the semiclassical approximation [5]. Within this approximation we have to separate the contribution arising from the condensate from the one of the other excited states, i.e.

$$
\theta_{\text {rig }}=m\left[\left\langle y^{2}+x^{2}\right\rangle_{0} N_{o}(T)+\left\langle y^{2}+x^{2}\right\rangle_{n c} N_{t h}\right]
$$

And

$$
\theta=m\left[\varepsilon_{0}^{2}\left\langle y^{2}+x^{2}\right\rangle_{0} N_{o}(T)+\left\langle y^{2}+x^{2}\right\rangle_{n c} N_{t h}\right]
$$

with $\left\langle y^{2}+x^{2}\right\rangle_{0}$ and $\left\langle y^{2}+x^{2}\right\rangle_{n c}$ are the condensate and non-condensate in situ radii in the redial direction respectively. The non- condensate atoms (thermal atoms) for ideal nonrotating atoms can be extracted from Eq.(11) and given by

$$
N_{\mathrm{o}}(T)=N-\left\{\left(\frac{K_{\beta} T}{\hbar \omega_{g}}\right)^{3} g_{3}(z)+\frac{3}{2}\left(\frac{\varpi}{w_{g}}\right)\left(\frac{K_{\beta} T}{\hbar \omega_{g}}\right)^{2} g_{2}(z)\right\}
$$

Where $\varepsilon_{0}$ is the deformation parameter of the condensate.

$$
\varepsilon_{0}=\frac{\left\langle x^{2}-y^{2}\right\rangle_{0}}{\left\langle x^{2}+y^{2}\right\rangle_{0}}
$$

However, this description is appropriate for temperatures $k_{D} T$ much larger than the oscillator energies $\hbar \omega_{x}$, $\hbar \omega_{y}$ and $\hbar \omega_{z}$ In Eq.(19), $\langle j|$ and $|i\rangle$ are eigenstates of the unperturbed Hamiltonian, $E_{i}$ and $E_{j}$ are the corresponding eigenvalues and $Z$ is the partition function. The indices \langle\rangle$_{0}$ and \langle\rangle$_{n c}$ in Eqs.(18) and (19) means that the average was taken over the densities of the Bose condensed and non condensed components in situ, respectively.

\section{In situ radii}

The square radii of the condensate boson, i.e. in situ radii, can be calculated using the HellmannFeynman theorem. This theorem relates the derivative of the energy eigenvalue with respect to a parameter, to the expectation value of the derivative of the Hamiltonian with respect to that same parameter,

$$
\frac{d \epsilon_{i}}{d \lambda}=\left\langle\psi(\lambda)\left|\frac{d H}{d \lambda}\right| \psi(\lambda)\right\rangle
$$

where $\epsilon_{i}$ is the energy eigenvalue for $H$. The parameter $\lambda$ can be any quantity that appears in

the Hamiltonian of the system, even physical constants such as $\hbar$.

$$
H=\frac{\mu}{2 m} \frac{1}{2} m \omega^{2} r^{2}
$$

With $p^{2}=p_{x}^{2}+p_{y}^{2}+p_{z}^{2}$. This Hamiltonian is characterized by a single particle energy eignvalue given by

$$
\epsilon_{i}=\left(i+\frac{3}{2}\right) \hbar \omega
$$

with $i$ is non-negative integer. For $\lambda=\omega$ Eq.(22) gives

$$
\left(i+\frac{1}{2}\right) \hbar=m \omega^{2}\left\langle r^{2}\right\rangle
$$

Thus the effective in situ radius of a single particle state $j n\rangle$ is given by its expectation value in this state, i.e.

$$
\left\langle r^{2}\right\rangle=\frac{\epsilon_{i}^{2}}{m \omega^{2}}
$$


However, the effective in situ radius of $N$ atoms can be parameterized from Bose-Einstein

Distribution $N=\sum_{i=0}^{\infty} \sum_{j=1}^{\infty} z^{j} e^{-j \beta \epsilon_{i}}$ Thus

$\left.\left.N\left\langle r^{2}\right\rangle=\sum_{j=1}^{\infty} z^{j} \sum_{i=0}^{\infty} \frac{\epsilon_{i}}{m \omega^{2}} e^{-j \beta \epsilon} \quad-\frac{1}{m \omega^{2}} \frac{\partial}{\partial \beta}\right)_{N, V} \sum_{j=1}^{\infty} \frac{z^{j}}{j} \sum_{i=0}^{\infty} e^{-j \beta \epsilon_{i}}=\frac{1}{m \omega^{2}} \frac{\partial q}{\partial \beta}\right)_{N, V}$

Finally, we reach to the main results of this

work. Substituting from Eq.(10), after setting

$\omega_{x}=\omega_{y}=\omega_{z}=\omega$ for non-rotating condensate we have

$$
\begin{gathered}
N\left\langle r^{2}\right\rangle=\frac{k_{\beta} T^{2}}{m \omega^{2}} \frac{\partial}{\partial T}\left[-\ln (1-Z)+\left(\frac{K_{\beta} T}{\hbar \omega}\right)^{3} g_{4}(z)+\frac{3}{2}\left(\frac{K_{\beta} T}{\hbar \omega}\right)^{2} g_{3}(z)\right] \\
=\frac{k_{\beta} T}{m \omega^{2}}\left\{\frac{-T}{z} \frac{\partial z}{\partial T} N_{0}(T)+3\left[g_{4}(z) \frac{\hbar \omega}{k_{\beta} T} g_{3}(z)\right]\left(\frac{K_{\beta} T}{\hbar \omega}\right)^{3}\right\}
\end{gathered}
$$

Here $q_{0}=-\ln (1-z) \quad, \quad$ and $N_{0}(T)=\frac{z}{1-z}$ are used here. The required

T-derivative of the fugacity $z$ above T0 can be obtained from Eq.(11),

$$
\frac{1}{z} \frac{\partial z}{\partial T}=-\frac{3}{T} \frac{g_{3}(z) \frac{\varpi}{\omega_{g}} \frac{T_{0}}{T}\left(\frac{\zeta(3)}{N}\right)^{1 / 3} g_{3}(z)}{g_{3}(z) \frac{3}{2} \frac{\varpi}{\omega_{g}} \frac{T_{0}}{T}\left(\frac{\zeta(3)}{N}\right)^{1 / 3} g_{1}(z)}
$$

Generalization of the above treatment to a trap with three different frequencies, $\left(\omega_{x} \omega_{y}\right.$ and $\left.\omega_{z}\right)$ is straightforward,

$$
\begin{gathered}
N\left\langle x^{2}\right\rangle=\left(\frac{k_{\beta} T}{m \omega_{g}^{2}}\right)\left(\frac{\omega_{y} \omega_{z}}{\omega_{x}^{2}}\right)^{\frac{1}{3}}\left\{\frac{-T}{z} \frac{\partial z}{\partial T} N_{0}(T)+3\left[g_{4}(z) \frac{\hbar \omega_{g}}{k_{\beta} T} \frac{\varpi}{\omega_{g}} g_{3}(z)\right]\left(\frac{K_{\beta} T}{\hbar \omega_{g}}\right)^{3}\right\} \\
N\left\langle y^{2}\right\rangle=\left(\frac{k_{\beta} T}{m \omega_{g}^{2}}\right)\left(\frac{\omega_{x} \omega_{z}}{\omega_{y}^{2}}\right)^{\frac{1}{3}}\left\{\frac{-T}{z} \frac{\partial z}{\partial T} N_{0}(T)+3\left[g_{4}(z) \frac{\hbar \omega_{g}}{k_{\beta} T} \frac{\varpi}{\omega_{g}} g_{3}(z)\right]\left(\frac{K_{\beta} T}{\hbar \omega_{g}}\right)^{3}\right\} \\
N\left\langle z^{2}\right\rangle=\left(\frac{k_{\beta} T}{m \omega_{g}^{2}}\right)\left(\frac{\omega_{x} \omega_{y}}{\omega_{z}^{2}}\right)^{\frac{1}{3}}\left\{\frac{-T}{z} \frac{\partial z}{\partial T} N_{0}(T)+3\left[g_{4}(z) \frac{\hbar \omega_{g}}{k_{\beta} T} \frac{\varpi}{\omega_{g}} g_{3}(z)\right]\left(\frac{K_{\beta} T}{\hbar \omega_{g}}\right)^{3}\right\}
\end{gathered}
$$

The first term in the curly brackets give the contribution arising from the particles in the condensate, while the second one is the contribution from the non condensed atoms. Result in Eq.(30) is a complementary to the
Stringari [5] results. In fact, this result constitute the main result which enables us to immediately calculate the finite size effect for the moment of inertia. Now we have

$\left\langle y^{2}+x^{2}\right\rangle_{n c} N_{t h}=3\left(\frac{k_{\beta} T}{m \omega_{g}^{2}}\right)\left[\left(\frac{\omega_{y} \omega_{z}}{\omega_{x}{ }^{2}}\right)^{\frac{1}{3}}+\left(\frac{\omega_{x} \omega_{z}}{\omega_{y}{ }^{2}}\right)^{\frac{1}{3}}\right]\left[g_{4}(z) \frac{\hbar \omega_{g}}{k_{\beta} T} \frac{\varpi}{\omega_{g}} g_{3}(z)\right]\left(\frac{K_{\beta} T}{\hbar \omega_{g}}\right)^{3}$

Finally, gathering Eqs.(18), (19), (20), (21) and (31) in terms of $\tau=\frac{T}{T_{o}}$ we have, 


$$
\begin{gathered}
\theta_{\text {rig }}=A\left\{\frac{-T}{z} \frac{\partial z}{\partial T}\left[1-\tau^{3}-\frac{3}{2} \frac{\varpi}{\omega_{g}} \frac{\zeta(2)}{\zeta(3)}\left(\frac{\zeta(3)}{N}\right)^{1 / 3} \tau^{2}\right]+\left[\frac{\zeta(4)}{\zeta(3)}+\frac{1}{\tau} \frac{\varpi}{\omega_{g}}\left(\frac{\zeta(3)}{N}\right)^{1 / 3}\right] \tau^{3}\right\} \\
\theta=A\left\{\varepsilon_{0}^{2} \frac{-T}{z} \frac{\partial z}{\partial T}\left[1-\tau^{3}-\frac{3}{2} \frac{\varpi}{\omega_{g}} \frac{\zeta(2)}{\zeta(3)}\left(\frac{\zeta(3)}{N}\right)^{1 / 3} \tau^{2}\right]+\left[\frac{\zeta(4)}{\zeta(3)}+\frac{1}{\tau} \frac{\varpi}{\omega_{g}}\left(\frac{\zeta(3)}{N}\right)^{1 / 3}\right] \tau^{3}\right\}
\end{gathered}
$$

Where

$$
A=3 N m\left(\frac{k_{\beta} T}{m \omega_{g}^{2}}\right)\left[\left(\frac{\omega_{y} \omega_{z}}{\omega_{x}^{2}}\right)^{\frac{1}{3}}+\left(\frac{\omega_{x} \omega_{z}}{\omega_{y}^{2}}\right)^{\frac{1}{3}}\right] \quad \varepsilon_{0}^{2}=\frac{\omega_{y}-\omega_{x}}{\omega_{y}+\omega_{x}}
$$

The physical interpretation of Eq. (33) is very clear. The first term in the moment of inertia arises from the atoms in the condensate, which contribute with their rotational flow and can hence be interpreted as the superfluid component. While the second term arises instead from the particles out of the condensate which rotate in a rigid way (normal fluid). These two distinct contributions are at the origin of an interesting $T$ dependence of $\theta$ Substituting Eqs.(32) and (33) in Eq.(14) we have,

$$
\frac{\rho_{S}}{\rho}=\frac{\left.\frac{-T \partial z}{z \partial T} \varepsilon_{0}^{2}\left[1-\tau^{3}-\frac{3 w \zeta(2)}{2 \omega_{g} \zeta(3)}\left(\frac{\zeta(3)}{N}\right)^{1 / 3} \tau^{2}\right]+\left[\frac{\zeta(4)}{\zeta(3)}+\frac{1 \omega}{\tau \omega_{g}}\left(\frac{\zeta(3)}{N}\right)^{1 / 3}\right] \tau^{3}\right\}}{\left.\frac{-T \partial z}{z \partial T}\left[1-\tau^{3}-\frac{3 \omega \zeta(2)}{2 \omega_{g} \zeta(3)}\left(\frac{\zeta(3)}{N}\right)^{1 / 3} \tau^{2}\right]+\left[\frac{\zeta(4)}{\zeta(3)}+\frac{1 \omega}{\tau \omega_{g}}\left(\frac{\zeta(3)}{N}\right)^{1 / 3}\right] \tau^{3}\right\}}
$$
This equation shows that, at $T>T_{0}$, i.
e $\left(\frac{\theta}{\theta_{\text {rig }}}\right)_{T}>T_{0}=1$, the superfluid fraction
equial to zero This behavior is in agreement with the experiment of liquid He II for which at temperature greater than $T_{\lambda}$ the liquid $\mathrm{He}$ is in the normal fluid phase. While at $T=0$ it reduce to the rotational form, where $\left(\frac{\theta}{\theta_{\text {ri }}}\right)_{T=0}=0$ , when the superfluid fraction approach unity, all the boson atoms are in the condensate.

The calculated .results from Eq.(35) are represented graphically in Figure (1). This figure shows that the superfluid fraction for a finite-sized

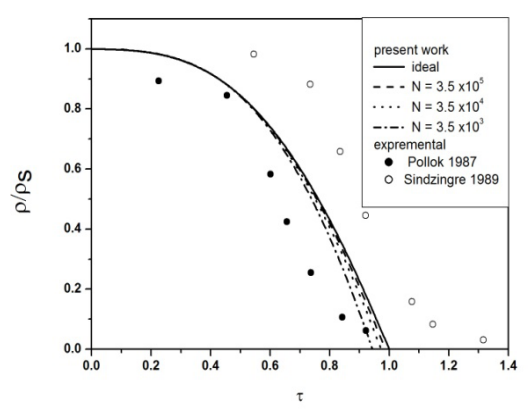

approaches unity at zero temperature. Moreover, the superfluid fraction goes smoothly to unity as temperature increases. These behavior is in agreement with the results of Sindzingre et ai. [22] and Pollock et al. [23]. As well as the superfluidity nature of the condensate bosons is a microscopic property that can be defined in a finite system or infinite system (the thermodynamic limit). Finite size effect is pronounced at temperature close to the transition temperature. Moreover leads to shift of the transition temperature. The effect of Bose statistics in a Bose liquid is to reduce the number of excited states and hence the coupling to an external potential. This can happen in a finite system As well as an infinite system

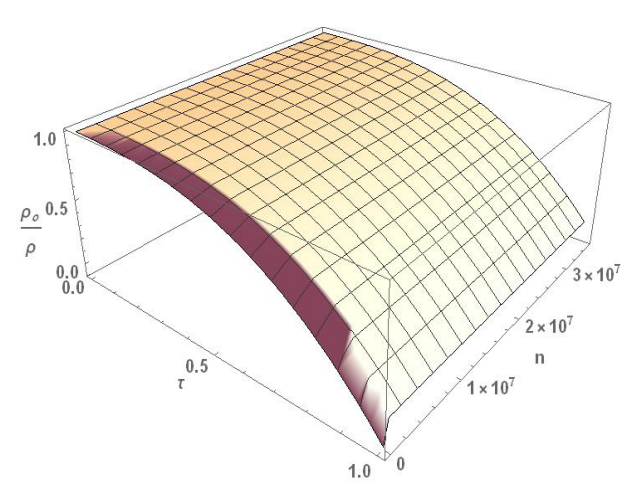

Fig.1. Superfluid fraction of an ideal Bose gas of finite size as a function of temperature The size is set by the number of particles. Black circles is the experimental data for [23] and open circles is the experimental data for Sindzinger [22]. 


\section{Heat capacity}

In superfluidity, measurement of the heat capacity have played an exceptionally important role in determining phase transitions and in characterizing the nature of bosonic excitations. We report on the calculation of the heat capacity for a rotating BEC .Unfortunately it is difficult to find a reliable analytical approximation for the heat capacity which allows to study whether it has a maximum value (peak) or not, and if it does, at what temperature it occurs. In the DOS approach, the heat capacity can be calculated by differentiating the internal energy $U$ with respect to the temperature, i.e. $C_{V}(T)=\left(\frac{\partial U}{\partial T}\right)_{N, V}$, with $U=K_{B} T^{2}\left(\frac{\partial q}{\partial T}\right)_{z}$. However, the treatment of the heat capacity is slightly more complicated because the energy has to be differentiated while keeping $N$, and $V$ fixed. Moreover, one has to take into account two different regimes, which are $T$ less or greater than $T_{0}$. For $T<T 0$, the heat capacity is given by

$$
\begin{aligned}
& \frac{C_{V}, T<T_{0}}{N K_{B}}=\frac{1}{\alpha}\left[12\left(\frac{T}{T_{0}}\right)^{3} \frac{\zeta(4)}{\zeta(3)}+9 \frac{3}{2} \frac{\varpi}{\omega_{g}}\left(\frac{\zeta(3)}{N}\right)^{1 / 3}\left(\frac{T}{T_{0}}\right)^{2}\right] \\
& \text { While for } \mathrm{T}>\mathrm{T}_{\mathrm{o}} \\
& \begin{aligned}
\frac{C_{V}, T>T_{0}}{N K_{B}}=\frac{1}{\alpha}[ & 12\left(\frac{T}{T_{0}}\right)^{3} \frac{g_{4}(z)}{\zeta(3)}+9 \frac{\varpi}{\omega_{g}} \frac{g_{3}(z)}{\zeta(3)}\left(\frac{\zeta(3)}{N}\right)^{1 / 3}\left(\frac{T}{T_{0}}\right)^{2} \\
+ & {\left.\left[3\left(\frac{T}{T_{0}}\right)^{4} \frac{g_{3}(z)}{\zeta(3)}+3 \frac{\varpi}{\omega_{g}}\left(\frac{T}{T_{0}}\right)^{3}\left(\frac{\zeta(3)}{N}\right)^{1 / 3} \frac{g_{2}(z)}{\zeta(3)}\right] \frac{T_{0}}{z} \frac{\partial z}{\partial T}\right] }
\end{aligned}
\end{aligned}
$$

We first recall that the temperature dependence of the heat capacity of bosons goes as $\left(T / T_{0}\right)^{\gamma}$ for $\epsilon^{1-\gamma}$ dependence of the DOS.

$$
\begin{gathered}
\frac{C_{V, T<T_{0}}^{\infty}}{N K_{B}}=\frac{12}{\alpha} \frac{\zeta(4)}{\zeta(3)}\left(\frac{T}{T_{0}}\right)^{3} \\
\frac{C_{V, T>T_{0}}^{\infty}}{N K_{B}}=\frac{3}{\alpha}\left[4 \frac{g_{4}(z)}{g_{3}(z)}-\frac{g_{3}(z)}{g_{2}(z)}\right]
\end{gathered}
$$

For non rotating condensation, i.e. $\alpha=0$ , (or $T=T_{o}$ ) the results previously obtained by Crossmann and Holthaus [18] are recovered. While in the thermodynamic limit $N \rightarrow \infty\left(R_{1}=0\right)$ Eq's .(36) and (37) are considerably simplify to,
It is clear that at, $T=T_{0}$ the heat capacity is discontinuous, there is a jump in the heat capacity.Moreover, using $C_{V}$ as an indicator, we can identified the magnitude of the jump and the order of the phase transition. The magnitude of the iumn increases with the rotation rate according to $\frac{6.577}{\alpha}$ This discontinuity characterizes the phase transition to be of second order according to the Ehrenfest definition. Furthermore, one observe that the heat capacity, Eq.(38), obeys the third law of thermodynamics, which demands a vanishing of the heat capacity at zero ( absolute) temperature and corresponds to the Dulnno-Petit lave in the very high temperature limit $\left(\frac{C_{V, T>T_{0}}}{N K_{B}}\right)_{T \rightarrow \infty}=3$

Keesom and Clusius [24] obtained data points which appeared to rise to a finite maximum value Egypt. J. Phys. Vol. 46 (2018) and then jump to a much lower value about one third that of the maximum [25]. They accordingly assigned a discontinuous curve to the data (see Figure 3). Because its shape resembled the Greek letter $\square$ this new transition was called the $\square$ transition. In Figures (2) and (3), a comparison of the temperature dependence of the heat capacity for rotating bosons in harmonic trap and the specific heat of superfluid He II is given. We claim that the qualitative correspondence between the curves for the BEC specific heat capacity and the specific heat experimental data of liquid ${ }^{4} \mathrm{He}$ are sufficient to suggest that BEC is a good simulator for the superfluid ${ }^{4} \mathrm{He}$. In this respect, the heat capacity of rotating condensate can be used to investigate the effect of finite size $N$ and rotation speed $\Omega$ on the superfluid He II 


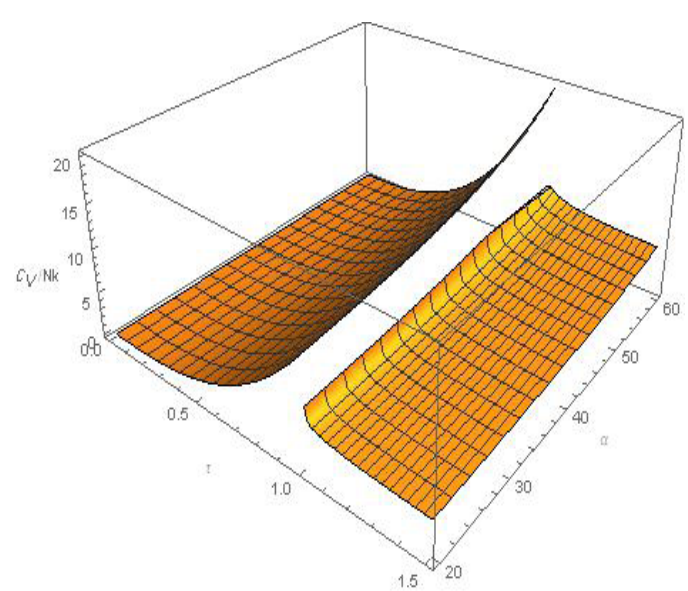

Fig. 2. The calculated. heat capacity The character of the lambda transition of heat capacity of BEC appear clearly.

Indeed, the results calculated from Eq's. (36) and (37) are represented in figure $(4,5)$ for different values of $N$ (finite size effect) and different values of $\Omega$ (rotation speed effect). The experimental values of Sindzinger et al. [22] and Pollock et al. [23] are shown for qualitatively comparison. This figures show that the BEC heat capacity is accompanied by a peak at the onset of the condensation, i.e. $T / T_{0}=1$. Increases of $N$ or $\Omega$ shifted to slightly lower temperature range than $T_{0}^{\prime} s$. Moreover, for $\tau=0, C_{V} / N K_{B}$ is

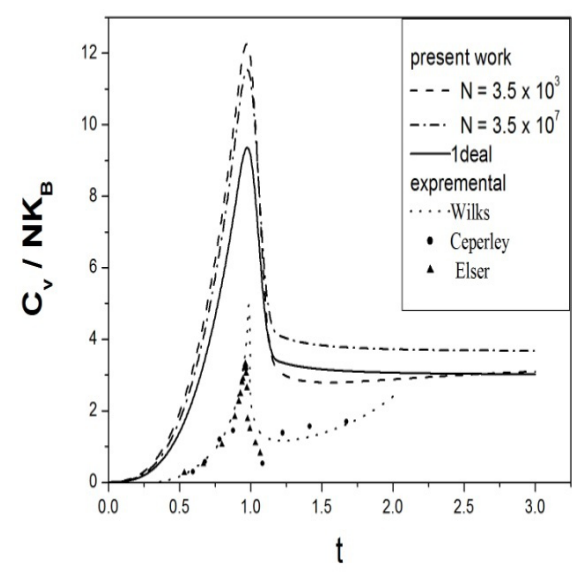

Fig. 4 . Relation between $C_{v} / N K_{B}$ and (Effect of finite size $\mathrm{N}$ in heat capacity )

shifted the transition temperature. The most remarkable point here is that, the heat capacity

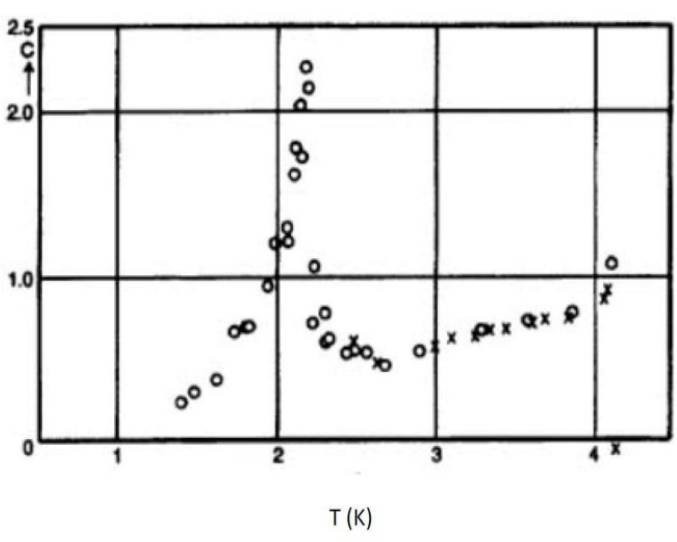

Fig. 3. The data of Keesom and co- workers from which heat capacity curves can be drawn.

vanishing, this behavior is agreement with the third law of thermodynamics. Increases $\tau$ leads to smoothly increasing nature for $C_{V} / N K_{B}$.

At $\tau={ }_{1} C_{V} / N K_{E_{6.57} \text { is }}$ iscontinuous, it has a jump, with magnitude $\frac{{ }^{6.577}}{\alpha}$ At $\tau>1, C_{V} / N K_{B}$ drops suddenly to its asymptotic value. The discontinuity at $\tau=1$ characterizes the phase transition to be 2 nd order. Finite size effect decreases the transition temperature. As well the rotation rate .

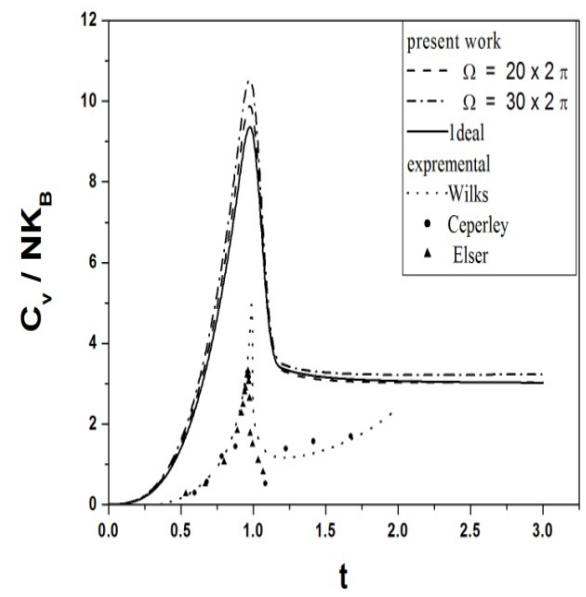

Fig. 5. relation between $C_{v} / N K_{B}$ and (Effect of rotation speed $\Omega$ in heat Capacity)

still have its ideal gas behavior. Thus it can be provided the same effects for the superfluid He II. 


\section{Conclusion}

In this work, we presented a theoretical investigation of the superfluidity of rotating alkali boson in harmonic trap as a simulator. The superfluid fraction and the heat capacity were calculated. We found that the system shows a qualitatively corresponds with the superfluid $\mathrm{He}$ II. In contrast to the complex microscopic structure of liquid helium, the simplicity of the theoretical description of dilute atomic gases allows a deep understanding of the basic mechanisms underlying superfluidity phenomena in simple terms. In conclusion, obviously, the phenomena of superfluid can be safely explained on the basis of the BEC phenomenon. At $T=0$,

BEC has a perfect superfluid behavior. Finite size effect leads to a shift for the $\square$ transition. This shift is propositional to $\frac{1}{N^{1 / 3}}$ Rotation effect leads to a shift for the $\square$-transition. This shift is propositional to $\frac{1}{\alpha}$, with $\alpha$ being the rotation rate. Finally, our method can be extended to investigate the superfluidity of interacting condensate boson.

\section{References}

1. Bloch, I., Dalibard, J. and Zwerger, W., Rev. Mod. Phys. 80, 885 (2008).

2. Cooper,N. R. and Hadzibabic, Z., Phys. Rev. Lett. 104, 030401 (2010); John, S. T., Hadzibabic, Z. and Cooper, N. R., Phys. Rev. A 83, 023610 (2011)

3. Andronikashvili, E. L., J. Phys. USSR 10, 201 (1946)

4. Ho, T. L. and Zhou ,Q., Nature Physics, 6, 131 (2009).

5. Stringari,S., Phys. Rev. Lett. 76, 1405 (1996).

6. Brosens, F.,Devreese, J. T. and Lemmens,L. F. , Phys. Rev. A 55, 2453 (1997).

7. Schneider,J. and Wallis, H. Eur. Phys. J. B 18, 507(2000)

8. Guery-Odelin, D. and Stringari S., Phys. Rev. Lett. 83, 4452 (1999)

9. Zambelli,F. and Stringarii,S.,Phys. Rev. A 63 33602 (2001)

10. Recatia,A., Zambellib, F. and Stringarib, S., Phys. Rev. Lett. 86, 377 (2001)
11. Van Druten, N. J., and Ketterle, W., Phys. Rev. Lett. 79, 549( 1997)

12. Kirsten,K. and Toms, D. J., Phys. Lett. A 222, 148 (1996) ; K. Kirsten, and D. J. Toms, Phys. Rev. A 54, 4188 (1996); K. Kirsten, and D. J. Toms , Phys. Lett. A 243, 137 (1998) ; K. Kirsten , and D. J. Toms , Phys. Rev. 59,158 (1999)

13. Hassan , A. S., El-Badry , A. M. and Soliman ,S. S. M., Eur. Phys. J. D 64, 465 (2011); ElBadry,A. M. andHassan, A. S., Eur. Phys. J. D 68, 76 (2014) ; Hassan,A. S., El-Badry, A. M. and Soliman, S. S. M., Eur. Phys. J. D 7 ( 2017) .

14. Grossmann, S.and Holthaus, M., Phys. Lett. A 208,188 (1995)

15. Cooper, N. R., Adv. Phys, 57, 539 (2008)

16. Stock,S., Battelier, B., Bretin , V., Hadzibabic, Z. and Dalibard, J., Laser Physics Lett. 2, 275 (2005) .

17. Fetter, A. L., Rev. of Mod. Phys. 81, 647 (2009)

18. Pathria, R. K., Statistical Mechanics (Pergammon, London, 1st ed. (1972)

19. Talbot ,E. F., Glyde , H. R., Stirling,W. G. and Svensson, E. C., Phys. Rev. B 38, 11229(1988)

20. Ali Shams, A. and Glyde, H. R., Phys. Rev. B 79, 214508 (2009)

21. Giorgini, F. D. S. , Pitaevskii, L. and Stringari, S., Rev. of Mod. Phys. 71, 463 (1999)

22. Sindzinger, P. , Klein, M. L. and Ceperley,D. M. , Phys. Rev. Lett. 63, 1601 (1989)

23. Pollock,E. L. and Ceperley,D. M. , Phys. Rev. B 36, 8343 (1987).

24. Keesom,W. H. and Clusius, K., Ueber die speci_sche $\mathrm{W} \sim$ arme des $\sim$ ussigen Heliums, Verhandlingen der Koninklijke Akademie van Wetenschappen (Amsterdam) 35, 307 (1932)

25. Jaeger,G., Arch. Hist. Exact Sci. 53, 51 (1998)

(Received:13/5/2018; accepted:27/5/2018) 
طبيعة السائل الفوقي لعدد محدد من تكثيفات بوز-اينشتين الدوارة علياء عادل محمود و محمود محمد صلاح وشيمي شعر اوي محمد سليمان قسم الفيزياء ـ كلية العلوم - جامعة المنيا - المنيا - مصر مصر مدري

تتطابق خواص عدد محدد من تكثيفات بوز - اينشتين الدوار مع خو اص السائل الفوقي للهيليوم ـ بناءا علي ذلك الكي

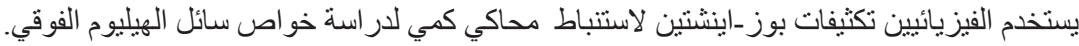

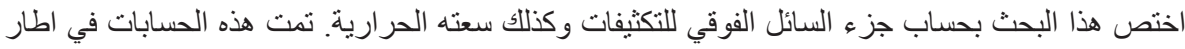

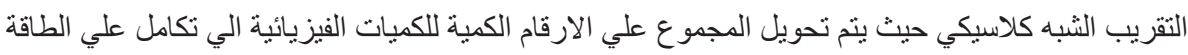

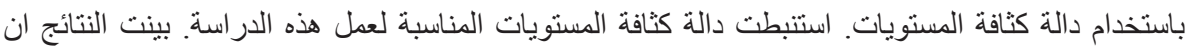

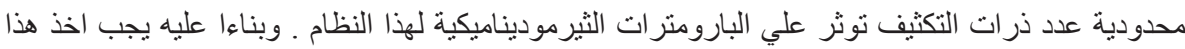
الثاثير عند اجراء اي قياس غير مباشر لهذا النظام (القياس الغير مباشر هو قيات الثياس كميات فيزيائية عمليا واستخدام

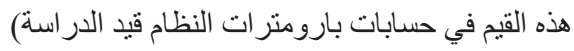

